P07.28 SYPHILIS TESTING IN ANTENATAL CARE: POLICIES AND PRACTICES AMONG LABORATORIES IN THE AMERICAS

${ }^{1} \mathrm{M}$ Luu, ${ }^{1} \mathrm{DC}$ Ham, ${ }^{1} \mathrm{ML} \mathrm{Kamb}{ }^{*},{ }^{2} \mathrm{~S}$ Caffe, ${ }^{3} \mathrm{KW}$ Hoover, ${ }^{2} \mathrm{~F}$ Perez. ${ }^{1}$ Division of STD Prevention, National Center for HIVIAIDS, Viral Hepatitis, STD and TB Prevention, Centers for Disease Control and Prevention (CDC), Atlanta, GA, USA; ${ }^{2} H I V$, Hepatitis, Tuberculosis and Sexually Transmitted Infections Unit, Communicable Diseases and Health Analysis Department, Pan American Health Organization (PAHO), Washington DC, USA; ${ }^{3}$ Division of HIVIAIDS Prevention, CDC, Atlanta, GA, USA

\subsection{6/sextrans-2015-052270.344}

Introduction The World Health Organization (WHO) recommends universal syphilis testing in pregnancy as part of basic maternal and child health services. Coupled with HIV testing, antenatal syphilis testing is fundamental for the regional initiative on elimination of mother-to-child transmission of HIV and congenital syphilis (EMTCT) in the Americas and globally. We conducted this survey of laboratory practices around syphilis testing to characterise syphilis testing in the Pan American Health Organisation (PAHO) member countries.

Methods A structured survey assessing syphilis laboratory testing practices in the 35 PAHO member states was administered electronically between March and August 2014. Leaders of national reference labs, large regional laboratories and a sample of local (e.g., large maternal hospitals, district hospitals) and private laboratories that conducted syphilis testing were recruited to participate. The survey collected data on laboratories tests used, testing algorithms applied in different clinical settings, testing volume and turnaround time, quality assurance strategies, and results reporting to national surveillance.

Results Data were obtained from 30 (86\%) PAHO member states, including 36 national or regional reference laboratories and 33 lower level laboratories, primarily (94\%) publicly funded. Of 69 laboratories reporting results, 41\% used rapid syphilis tests (RSTs), of which 36\% were lower level laboratories. Sixty-eight percent of reporting laboratories $(83 \%$ of national/ regional) participated in external quality control, and $36 \%$ reported surveillance data. Of the 69 laboratories, 49 (71\%) reported using a national algorithm for syphilis testing in pregnancy, of which 5 involved RSTs. Of 54 (78\%) laboratories that reported processing samples from antenatal clinics, approximately half experienced stock outs of at least one essential commodity during the previous 12 months.

Conclusions Updating laboratory algorithms along with improving testing standards and quality assurance, supply distribution, and surveillance reporting could better advance EMTCT of syphilis and improve syphilis testing in various clinical settings in the Americas Region.

Disclosure of interest No grants or outside funding were received in the development of this study.

\section{P07.29 POINT OF CARE TESTING (POCT) FOR HIV; CAN VENIPUNCTURE BE AVOIDED FOR SYPHILIS?}

${ }^{1}$ MA O'Reilly*, ${ }^{2} \mathrm{~N}$ Ryder. ${ }^{1}$ Pacific Clinic, Sexual Health Services HNE Local Health District NSW; ${ }^{2}$ ACON Clinical NSW

\subsection{6/sextrans-2015-052270.345}

Introduction Point of Care Testing (POCT) for HIV potentially removes the need for venipuncture; however it is currently still required for syphilis and hepatitis B testing. Syphilis POCTs exist but most cannot be used for clients with a past history of syphilis. Newcastle Sexual Health Service explored whether venipuncture could be avoided at the Hunter ACON outreach clinic. Methods A list of MSM seen at Hunter ACON during 2014 was obtained from the clinic database. Data for the following parameters were obtained from laboratory and clinic records: numbers of individual clients, occasions of service, if they were first time presenters to the service, past history of syphilis and HBV status. Results 105 MSM clients were tested for syphilis on 150 occasions during 2014 at Hunter ACON with 2 clients testing positive on the syphilis screening EIA. Both clients were known to have been previously diagnosed with syphilis. 71 clients attended as a first visit, 40 of these were of unknown hepatitis A or B status and hence may have required HBV testing. Further analysis of reported HBV status may reduce this number as NSW has been vaccinating MSM and school aged males for many years. Overall 108 of 150 (72\%) HIV testing occasions could have avoided venipuncture through use of HIV and syphilis rapid testing.

Conclusion This data demonstrates that a syphilis POCT could be used for the overwhelming majority of MSM seen at the Hunter ACON outreach clinic. In at least $72 \%$ of occasion's venipuncture could be avoided when performing HIV/STI testing at the Hunter ACON clinic.

Disclosure of interest statement N/A.

\section{P07.30 IMPACT OF EXPANDED SCREENING ON THE DETECTION OF HIV AND SYPHILIS IN WUXI, CHINA}

${ }^{1} \mathrm{XJ}$ Meng ${ }^{*},{ }^{2} \mathrm{HC}$ Zou. ${ }^{1}$ Wuxi Center for Disease Control and Prevention, Wuxi, China; ${ }^{2}$ Kirby Institute, University of New South Wales, Australia

\subsection{6/sextrans-2015-052270.346}

Introduction HIV and syphilis shares same mode of transmission. In 2010 the Chinese government adopted expanded HIV and syphilis screening strategy (EHSS) across the country in order to timely detect people with these two infections. The impact of this strategy has not been well documented.

Methods HIV and syphilis surveillance data 2004-2014 in Wuxi, China were retrieved. Sources of surveillance data included general hospitals (GHs), sexual health clinics (SHCs), blood donation centres (BDCs), voluntary counselling and testing clinics (VCTs) and others in Wuxi. We used Poisson distribution events test to compare number of HIV and syphilis testing, Chi-squared test to compare HIV and syphilis positive rates and proportions of source of HIV and syphilis notification, between the period before EHSS (Period I, 2004-2009) and the period after EHSS (Period II, 2010-2014).

Results Comparing Periods I and II, 586,000 vs $1,423,000$ person-times were screened for both HIV and syphilis $(\mathrm{P}<0.001)$; HIV positive rates were $0.08 \% \quad(476)$ vs $0.13 \% \quad(1,854)$ $(<0.001)$; syphilis positive rates were $0.37 \%(2,172)$ vs $0.63 \%$ $(8,955)(\mathrm{P}<0.001)$. In Period I, 18.8\%, 10.9\%, 7.1\%, 14.2\% and $49.0 \%$ of all HIV positive cases were from GHs, SHCs, BDCs, VCTs and other sources, respectively. This compared to $25.8 \%, 7.4 \%, 9.0 \%, 16.5 \%$ and $41.3 \%$ in Period II ( $\mathrm{P}<0.001)$. In Period I, $42.0 \%, 13.7 \%, 7.8 \%, 1.5 \%$ and $35.0 \%$ of all syphilis positive cases were from GHs, SHCs, BDCs, VCTs and other sources, respectively. This compared to $26.7 \%, 28.0 \%, 17.7 \%$, $1.7 \%$ and $25.9 \%$ in Period II $(\mathrm{P}<0.001)$.

Conclusion Both the number of HIV and syphilis testing and positive rate increased as a result of EHSS. More HIV infections were detected from GHs compared to syphilis from SHCs in 